\title{
A Unified Solution for Gateway and In-Network Traffic Load Balancing in Multihop Data Collection Scenarios
}

\author{
Ye Miao, Serdar Vural, Zhili Sun, and Ning Wang
}

\begin{abstract}
Data collection is a fundamental task of wireless sensor networks to support a variety of applications, such as remote monitoring, and emergency response, where collected information is relayed to an infrastructure network via packet gateways for processing and decision making. In large-scale monitoring scenarios, data packets need to be relayed over multihop paths to the gateways, and sensors are often randomly deployed, causing local node density differences. As a result, imbalance in data traffic load on the gateways is likely to occur. Furthermore, due to dynamic network conditions and differences in sensor data generation rates, congestion on some data paths is also often experienced. Numerous studies have focused on the problem of in-network traffic load balancing, whereas a few works have aimed at equalizing the loads on gateways. However, there is a potential tradeoff between these two problems. In this paper, the dual objective of gateway and in-network load balancing is addressed, and the reactive and adaptive load-balancing (RALB) algorithm is presented. RALB is proposed as a generic solution for multihop networks and mesh topologies, particularly in large-scale remote monitoring scenarios, to balance traffic loads.
\end{abstract}

Index Terms-Data collection, load balancing, mesh networks, multihop wireless sensor networks (WSNs), remote monitoring.

\section{INTRODUCTION}

W IRELESS Sensor Networks (WSNs) support a variety of data collection applications, such as environmental monitoring [1], [2], tactical military monitoring [3], traffic surveillance [4], video surveillance, and security [5]. Environmental/ ambient monitoring applications have become particularly popular, and numerous systems have been deployed to observe some metrics, such as atmospheric conditions [6], noise levels [7], and soil and water quality [8]. Delivery of event-triggered or periodically collected data is performed by a deployed WSN, in which sensor nodes are data collection units. To ensure the success of these applications, it is of utmost importance to have accuracy and reliability at all system parts, which are: 1) measurement devices, i.e., sensors; 2 ) a data delivery component, i.e., a WSN; and 3) a head-end system that performs automation, control, and analysis.

Manuscript received October 20, 2014; revised June 3, 2015; accepted July 9, 2015. This work was supported by the Chinese Academy of Telecommunications Technology under Grant 2007 DQ305156.

The authors are with the Institute for Communication Systems (ICS), University of Surrey, Guildford GU2 7XH, U.K. (e-mail: y.miao@surrey.ac.uk; s.vural@surrey.ac.uk; z.sun@surrey.ac.uk; n.wang@surrey.ac.uk).

Digital Object Identifier 10.1109/JSYST.2015.2457674
In recent projects, efforts have been made to enhance the quality of collected data by providing better measurement techniques, automation processes, and systems. For instance, the Cool It! project [6] has a systems focus, which aims to provide an accurate representation of global atmospheric conditions toward estimating future temperature and humidity levels at specified locations. Similarly, Advanced Technologies for Water Resource Management (ATWARM) [9] provides an automated monitoring platform for water and waste-water treatment processes in remote locations. In ATWARM, the quality and overall cost of measurement is improved by applying a microfluidic technology. Another example is SENSEIVER [8], which has developed a data acquisition system that allows storage, processing, and real-time analysis of air, soil, and water conditions in order to evaluate pollution levels. The project has developed cost-efficient sensors and energy-efficient transmitters, new materials that improve measurement quality, and data acquisition boards and sensor modules.

Aside from the recent advances in sensing technology, system architecture, and automation systems, efficiency and reliability in delivery of collected data from the area of observation to the head-end control center is also crucial for environmental monitoring applications. Satellite systems are typically used, providing global connectivity to a remote observation field without the need for an additional end-to-end infrastructure [10], [11]. Portable satellite terminals can easily and quickly be deployed in remote and even hazardous environments. To achieve effective and efficient data delivery in remote monitoring applications, recent projects have focused on satellitebased connectivity. For instance, MONET [10] has developed solutions for integrating satellite networks and mobile ad hoc networks in forest fire and mountain rescue scenarios. The ARGOS project [12] has focused on wildlife monitoring, oceanography, climatology, marine surveillance, and offshore monitoring scenarios.

\section{A. Problem Statement}

In remote environmental monitoring applications via satellite systems, a typical problem is the low data rate supported by the satellite link, e.g., the widely used Inmarsat Broadband Global Area Network portable terminals allow uplink bit rates of 4.5 to $492 \mathrm{~kb} / \mathrm{s}$ [13]. For example, in MONET project [10], the aggregate data rate generated by network nodes can exceed this maximum rate of $492 \mathrm{~kb} / \mathrm{s}$, where nodes send data and images of $1-\mathrm{Mb}$ average size and set up visioconference sessions of 
$256 \mathrm{~kb} / \mathrm{s}$ periodically. Considering the sensing data rate for a typical sensor within a range $10-250 \mathrm{~kb} / \mathrm{s}$ [14], [15], simultaneous sensory data can itself generate traffic peaks. To support the traffic load and also ensure quick data delivery, multiple terminals are typically deployed in the area of observation, where they act as packet gateways ${ }^{1}$ to the satellite network and as data sinks for the WSN that collects the data. However, when part of the generated traffic is in closer proximity to a particular satellite terminal than others, the bulk of the data is likely to be directed to that terminal, as conventional routing algorithms favor shortest paths [16].

Hence, the data transferred from the WSN to the satellite network may create disproportionate traffic loads on the network's data sinks [17], [18], which may cause some of the gateways to drop data packets or delay packet transmissions.

In addition to the potential load imbalance problems across the network's gateways, data traffic within the network may have high variation on data delivery paths. This is caused by the differences in the proximity between gateways and the locations where newly sensed events emerge, as well as the node density at different areas of the network (often caused by random node deployment) [19], [20]. Numerous studies have addressed this problem of in-network traffic imbalance from various perspectives [19]-[28], with the common aim of reducing traffic congestion. However, even if the traffic is fairly balanced among network paths, any overloaded data sinks may diversely impact the total data throughput to the infrastructure. As a remedy, some studies [17], [18] propose to divert the traffic from overloaded gateways to underused ones; however, this traffic migration itself may create new congested paths, leading to packet drops and eventually low throughput. In short, there is a tradeoff between balancing the traffic within the network and equalizing gateway traffic loads, particularly when the data sinks are at far away locations.

\section{B. Our Contribution}

In this paper, the dual objective of gateway and in-network load balancing is addressed, and the reactive and adaptive load balancing (RALB) algorithm is proposed as a generic solution for any multihop wired/wireless network with multiple data gateways connecting the network to an infrastructure. There are two goals of RALB: 1) To equalize gateway utilization levels as much as possible; and 2) to balance the traffic load on the paths to the gateways. RALB can strike the balance between these potentially conflicting objectives by reducing the standard deviation of gateway utilization while avoiding in-network congestion. A distributed path selection algorithm helps select the least loaded paths via independent routing decisions made by intermediate nodes on data paths. To avoid route flapping [29] problems, probabilistic decision thresholds are set, which are used to limit the frequency of route changes in an adaptive way according to link and traffic conditions.

The remainder of this paper is organized as follows. Section II presents the literature review of the related work on load balancing in multihop wireless networks. Section III

${ }^{1}$ The terms "gateway" and "data sink" are used interchangeably in this paper. illustrates concepts and explains RALB in detail. Simulation results and performance analysis are presented in Section IV. Finally, Section V concludes this paper.

\section{RELATED WORK}

Existing studies on traffic load balancing in multihop wireless networks can be broadly categorized into three groups, based on their load-balancing goal: 1) in-network load balancing; 2) gateway load balancing; and 3) joint in-network and gateway load balancing.

\section{A. In-Network Load Balancing}

The studies in this category propose various path quality metrics, and aim to obtain an even and fair distribution of the collected data traffic among the set of available paths. A balanced traffic load within the network may create congested links, which causes packet drops, excessive packet retransmissions, and hence low network throughput. For instance, Mhatre et al. [24] propose a load-balancing algorithm based on the ETP (expected throughput [25]) routing metric, which takes into account the capacity reduction of a link due to its interaction with other links within its contention domain. In [26], the contention level, congestion level, and hop distance are combined as the selection metric to avoid areas with high data traffic or channel contention. Similarly, the algorithm in [27] combines multiple network performance metrics, including path availability period, residual link capacity, and latency. Jung et al. [28] proposed a routing algorithm that considers hop distance to gateways and traffic volume at each node. Although these (and many similar other) algorithms achieve a certain level of in-network traffic load balance, the capacity (processing speed and transmission bandwidth) of data sinks are not considered as an algorithm parameter. Hence, such algorithms are not suitable for the data collection scenarios, in which multiple data sinks act as packet gateways and forward data traffic to another network. Some gateways can easily be overloaded if gateway capacities are not considered.

\section{B. Gateway Load Balancing}

Some solutions are proposed to evenly distribute the forwarded traffic on the network's exit points, i.e., the gateways; however, without sufficient consideration of the in-network traffic dynamics. The greedy selection of the least loaded gateways gives rise to overloading of some critically located nodes where traffic flows merge, turning them into traffic bottlenecks. Furthermore, route flapping [29] is a common issue. Most solutions in this category model a gateway's load using different gateway parameters, such as total traffic [30], residual capacity [31]-[33], number of active flows [17], average queue length [34], and number of registered mobile nodes [35].

\section{Joint In-Network and Gateway Load Balancing}

There are only a few studies that aim to balance the traffic load over the network, while also taking certain measures to 
avoid overloading the network's gateways. For instance, in [36], gateway load is considered in conjunction with two path metrics, route interference and expected link quality, to form a single metric. This study uses a proactive approach, in which gateways periodically broadcast advertisement packets to notify network nodes of their residual capacity and mobile nodes periodically broadcast probe packets to exchange information with their neighbors on packet delivery ratio and the level of channel interference. Such proactive approaches have high control message overhead.

In [37], a source-based gateway selection scheme is proposed, which also combines path metrics and gateway load into a single metric. Different to [36], nodes do not immediately switch routing paths after discovering better alternatives, but wait for a random time period. This is a measure to prevent nodes from simultaneously changing their routing paths and to have more stable routes. Furthermore, path rankings may also change during such waiting periods due to network dynamics, which actually may lead to selection of worse paths. Finally, the path selection algorithm runs only at data sources; in fact, intermediate nodes, which forward data packets must ideally also take part in path selection, making it a distributed algorithm that can better adapt to network dynamics.

A hybrid path selection metric is proposed in [38], which is a combination of intra-MANET ${ }^{2}$ traffic within a MANET domain, and inter-MANET traffic over multiple gateway domains. The metric is a linear combination of three components: shortest path distance (hop count), inter-MANET traffic load (modeled as the total number of registered nodes on gateways), and intra-MANET traffic load within each gateway's domain (node density). However, this paper focuses on network performance parameters (packet delivery ratio, signaling overhead, and packet transmission delay) and does not provide loadbalancing results.

Galvez et al. [18] propose a fast greedy algorithm to equalize the load of gateways while also avoiding gateway flapping. Mobile nodes are first ordered in ascending order based on the number of valid paths/gateways, and then assigned to the best valid paths. The valid paths are ranked based on the gateway load (number of flows the gateway serves) and path cost (expected interference and hop distance). However, decisions on gateway-node associations are made in a centralized controller, which is linked to the gateways by wired connections. Centralized solutions are likely to determine the most suitable gateway-node associations; however, in networks with dynamically changing conditions, the control message overhead necessary for capturing a global view of the network at a single location is significant [39].

One common drawback of existing schemes in this category is that the gateway load-balancing target is the equalization of traffic load among gateways. In fact, what is more important is the utilization of a gateway's capacity, rather than the absolute value of its traffic load. This is highlighted in Section III-B2, where gateway utilization is formally defined, and proposed as an added value of RALB.

\footnotetext{
${ }^{2}$ MANET: Mobile ad hoc network.
}

\section{REACTIVE AND AdAPTIVE LOAD BALANCING (RALB)}

The proposed solution for the dual objective of gateway and in-network load balancing is based on four principles:

1) Dynamic and reactive discovery of multiple paths at each node to the set of gateways $G$, which connect the network to an infrastructure,

2) No central point of decision making, no collection of global network information, and no periodic networkwide packet broadcasts,

3) Dynamically adapted node-centric parameters to avoid frequent route switches,

4) Reactive and timely update of path and gateway metrics, as part of path discovery, with minimal additional control overhead.

To achieve reactive path discovery, the basic requirement of RALB is the availability of two packet types [40]:

1) Route Request (RREQ): Generated by a data source node to its neighbors when the node does not have any nexthop node information (node ID or network address) in its routing table, corresponding to the desired destination node. RREQ packets are forwarded by each intermediate node if no next-hop node information is available, until reaching the destination.

2) Route Reply (RREP): Generated as a response to a received RREQ packet when $(i)$ the node is an intermediate node and has a fresh entry in its routing table for the requested destination, or (ii) the node is the destination itself. The RREP packet follows the reverse path that the corresponding RREQ packet has followed, until reaching the source node that originally generated the RREQ.

\section{A. RALB: A Load-Balancing Solution for Multipath Reactive Routing Protocols}

On-demand routing protocols are available in the current literature, such as dynamic source routing [41] and ad hoc ondemand distance vector (AODV) routing [40], which use the two basic packet types, i.e., RREQ and RREP, for route discovery. AODV is a routing protocol used by ZigBee technology [42] for mesh deployments of WSNs [43], [44]. Such protocols provide a single path to each destination node. Instead, a later proposed protocol, called the ad hoc on-demand multipath distance vector (AOMDV) routing [16], discovers multiple loopfree and disjoint paths to each destination node in order to improve reliability and reduce end-to-end (E2E) time delay, as compared with AODV. In fact, by reducing the frequency of route updates, AOMDV achieves around 30\% saving in control overhead, as compared with the single-path protocol AODV. This makes AOMDV a more energy-efficient routing protocol for resource-limited WSNs.

RALB is a load-balancing solution that aids routing decisions and is therefore independent from the particular choice of routing protocol, as long as the protocol is reactive and provides multiple paths to each gateway. AOMDV is chosen as a baseline reactive routing protocol, which can provide network nodes with multiple paths to each gateway. 


\section{B. Path Quality Metric of RALB}

Each node that is engaged in data communication (data source or forwarding node) assigns a quality metric to each path it discovers. This path quality metric, which is denoted by $q$, is formed of multiple performance metrics. Here, the definitions of these metrics are provided, before describing the details of RALB.

The path quality metric $q(g, i)$ for a path $i$ from a node to a gateway $g$ is designed in a way to capture two factors that affect a path's overall quality: 1 ) packet delivery performance $q_{d}(g, i)$ to gateway $g$ from the node; and 2) the capability of gateway $g$ to service its incoming data traffic, which is denoted by $q_{c}(g)$. Hence

$$
q(g, i)=\beta q_{c}(g)+(1-\beta) q_{d}(g, i)
$$

where $0<\beta<1$ is a weighting factor. ${ }^{3}$ The coefficient $\beta$ captures the desired level of emphasis given (by the network operator or the application service) to two potentially conflicting goals:

1) Choosing a gateway that is more capable of relaying its incoming traffic to the outside network,

2) Choosing a path that has higher performance in delivering data traffic to a gateway.

The packet delivery performance $q_{d}(g, i)$ is modeled by a weighted sum of normalized path metrics, namely residual bandwidth, path delay, and path reliability, whereas gateway capability $q_{c}(g)$ is modeled by a single metric called gateway utilization. These metrics are discovered/updated as part of the reactive path discovery, i.e., carried over multihop paths from gateways to data sources in RREP packets, as explained next.

1) Packet Delivery Performance Metrics of a Path:

a) Residual path bandwidth: This is the currently available bandwidth on a data path between a source sensor and a gateway, when a path selection decision is being made at the sensor. The residual bandwidth on a path $i$ to a gateway $g$ is denoted by $b(g, i)$.

Since a path is a sequence of links, residual path bandwidth is considered as the minimum of the residual link bandwidths over all the links along that path. It also includes the gateway's capability to handle incoming data traffic, which is affected by its data processing speed, as well as the residual bandwidth on its link to the infrastructure network.

Residual link bandwidth is considered as the maximum transmission rate ${ }^{4}$ that a transmitter can inject packets to the link. As such, auto-rate adaptation [45] is used so that nodes select the highest transmission rate, which constitutes the link's residual bandwidth. This is a practical and precise method, as opposed to modeling residual link bandwidth simply as the difference between link capacity and current traffic load [27].

Denoting the link residual bandwidth from a node $A$ to its neighbor $B$ as $b(A \rightarrow B)$, and the residual path bandwidth from node $B$ to gateway $g$ (reported by $B$ to $A$ in the route reply)

\footnotetext{
${ }^{3}$ Alternative methods of combing these two factors can be utilized.

${ }^{4}$ Maximum transmission rate is a random variable, which is a result of two main factors: the link's capacity, and the current level of channel interference.
}

as $b(B \rightarrow g)$, $A$ 's residual path bandwidth $b(g, i)$ on path $i$ through $B$ to $g$ is

$$
b(g, i)=\min (b(A \rightarrow B), b(B \rightarrow g)) .
$$

b) Path latency: This is the total end-to-end time delay over a path, which consists of queuing, processing, transmission, and propagation delays. The latency of a path $i$ that leads to a gateway $g$ is denoted by $l(g, i)$. As explained in Section III-A, nodes discover paths via RREQ packets sent/forwarded to the gateways (as in AOMDV [16]). Nodes note the time instance when they transmit a RREQ packet toward gateway $g$. The node that replies the RREQ with a RREP, for example, $x$, includes two pieces of information in the RREP packet: 1) its own latency $l$ to the gateway, which is denoted by $l(x \rightarrow g)$; and 2$)$ the time instance when the RREP is transmitted back. Upon reception of the RREP, each receiving node calculates the time period $t$ that it takes for the RREQ to reach the replying node $x$, and updates its latency to gateway $g$ on path $i$ as

$$
l(g, i)=l(x \rightarrow g)+t .
$$

This is used as a practical way to monitor path latency to gateways by every source as well as every node that participates in packet forwarding.

c) Reliability: This is defined as the ratio of successful end-to-end packet delivery over a path, and is denoted by $0 \leq r(g, i) \leq 1$ for a path $i$ leading to a gateway $g$. Periodic neighborhood messages are exchanged between nodes, which act as probe packets. By monitoring the delivery ratio of these messages, nodes keep track of the reliability of their links to their neighbors. Then, a path's reliability (from a node to the gateway) is computed by the product of the packet delivery ratios of all the links forming that path.

Link reliability values are delivered to nodes via RREP messages, as part of RALB's path discovery (see Section III-A). Accordingly, a node $A$ calculates the reliability $r(g, i)$ of a path $i$ to a gateway $g$ through its neighbor $B$, as a product of the path reliability $r(B \rightarrow g)$ from $B$ to $g$ (reported in the route RREP), and the reliability $r(A \rightarrow B)$ of its link to $B$

$$
r(g, i)=r(A \rightarrow B) \cdot r(B \rightarrow g) .
$$

2) Gateway Utilization: Utilization $U_{g}$ of a gateway $g$ is defined as the ratio of the data traffic load on the gateway to its capacity $C_{g}$, where $C_{g}$ is the minimum of: 1 ) the residual bandwidth of the gateway's outgoing link to the infrastructure network; and 2) the gateway's data processing speed.

Gateway utilization is the preferred metric to represent a gateway's capability to service its incoming data traffic load, since different gateways may have different properties, i.e., processing speed, bandwidth on outgoing link (instead of the traffic load itself, as in previous studies [30]-[33]). This concept is illustrated in Fig. 1(a) and (b), for traffic load and gateway utilization, respectively, when chosen as the metric to represent a gateway's service capability. As shown in Fig. 1(a), the gateway with the lower capacity is critically loaded when the traffic loads are equalized; whereas in Fig. 1(b) this gateway 


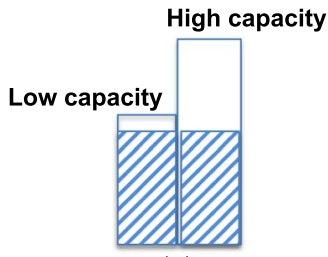

(a) (b)
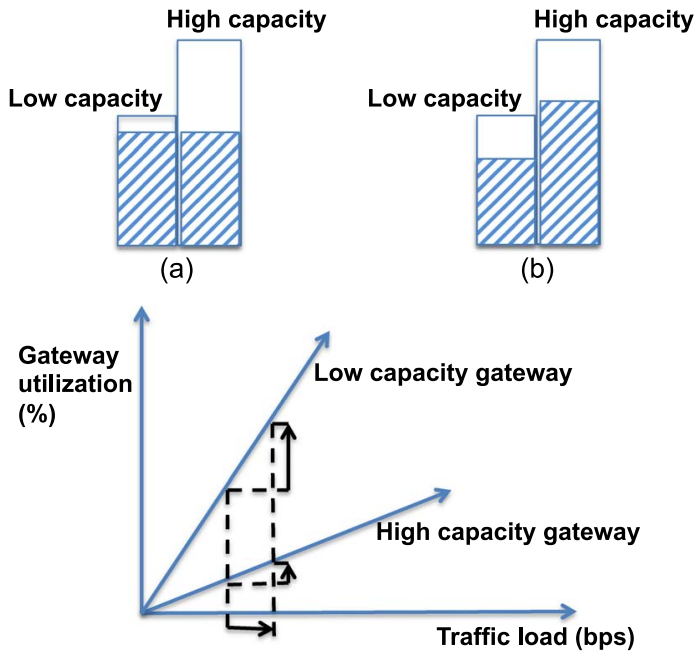

(c)

Fig. 1. Metric choice for gateway load balancing. Traffic load equalization versus gateway utilization equalization.

is in a better state when gateway utilizations are equalized. In fact, as shown in Fig. 1(c), a certain amount of increase in traffic load affects the gateway with the lower capacity more critically, via increasing its utilization by a larger amount. This shows that the aim of gateway load balancing must be to equalize utilization levels rather than the traffic loads that different gateways receive.

3) Path Quality Metric: To combine the path delivery performance metrics into a single path quality metric, first, the following normalization operations are performed, so that unitless quantities that fall in the same value range of $(0,1]$ are obtained

$$
\begin{aligned}
q_{d}^{b}(g, i) & =\frac{b(g, i)}{\max _{j \in P_{g}} b(g, j)}, q_{d}^{l}(g, i)=1-\frac{l(g, i)}{\max _{j \in P_{g}} l(g, j)} \\
q_{d}^{r}(g, i) & =\frac{r(g, i)}{\max _{j \in P_{g}} r(g, j)}
\end{aligned}
$$

where $q_{d}^{b}(g, i), q_{d}^{l}(g, i)$, and $q_{d}^{r}(g, i)$ denote the normalized bandwidth, latency, and reliability metrics of path $i$ 's delivery performance to a gateway $g$, respectively, and $P_{g}$ denotes the set of paths to gateway $g$.

The normalized metrics in (5) are combined as a weighted sum, which represents the path quality metric of path $i$ to gateway $g$, given by

$$
q_{d}(g, i)=\alpha_{b} q_{d}^{b}(g, i)+\alpha_{l} q_{d}^{l}(g, i)+\alpha_{r} q_{d}^{r}(g, i)
$$

where $\alpha_{b}+\alpha_{l}+\alpha_{r}=1$. Accordingly, a higher path quality metric is obtained if a path has higher reliability, higher bandwidth, and less latency. The coefficients $\alpha_{b}, \alpha_{l}$, and $\alpha_{r}$ are directly related to the quality-of-service (QoS) requirements of different applications, e.g., an application with stringent time delay limits would choose a large $\alpha_{l}$, whereas an application that must support high bit rates would pick a large $\alpha_{b}$. These coefficients represent the QoS settings used for different flows; hence the set of coefficients are likely to be different for flows of different QoS classes; however, they are kept constant for all packets that belong to the same flow.
TABLE I

RALB ROUTING TABLE ENTRY

\begin{tabular}{|c|c|c|c|c|c|c|}
\hline $\begin{array}{c}\text { Gateway } \\
\text { ID }\end{array}$ & $\begin{array}{c}\text { Next } \\
\text { hop ID }\end{array}$ & $\begin{array}{c}\text { Path quality } \\
\text { metric }\end{array}$ & Bandwidth & Latency & Reliability & $\begin{array}{c}\text { Gateway } \\
\text { utilization }\end{array}$ \\
\hline
\end{tabular}

\section{Update of the Path Quality Metric and the Routing Table}

Upon reception of an RREP packet, each node checks the reported path metric values, and updates them considering the link conditions to the next hop node from where the packet is received, according to (2)-(4). Then, using the reported gateway utilization $U_{g}$ in the RREP and these updated path metrics, the path quality metric is calculated by (5) and (6). The routing table corresponding to the path is updated if necessary, i.e., in case of a new entry or a higher metric value. The routing table entry in RALB is shown in Table I.

When a new packet arrives, if multiple paths are available for the destination gateway, then RALB runs a path-gateway selection algorithm to decide the next hop to where the packet is to be forwarded. This is explained next.

\section{Gateway and Path Selection}

Here, the path and gateway selection algorithm provided by RALB is described. RALB runs at every network node and assists the underlying multipath reactive routing protocol (AOMDV, in this case) that is normally load unaware. This is achieved by selecting the best available path $p^{*}$ to each gateway, according to the path quality metric $q$ as introduced in (1), i.e., the path $p^{*}$ with the highest metric value $q\left(p^{*}\right)$. However, the originally selected path $p_{o}$ by the load-unaware routing protocol (AOMDV) may be different $\left(p^{*} \neq p_{o}\right)$, and $q\left(p^{*}\right) \neq q\left(p_{o}\right)$. For instance, AOMDV replaces the existing paths with the newly discovered ones and ranks the set of its available paths in terms of their E2E time delays, where the path with the least time delay is regarded as the best one. In this paper, changing from the shortest path $p_{o}$ to RALB's highest metric path $p^{*}$ is referred to as a path switch.

A timely made path switch decision may help remove innetwork congestion and/or lower the traffic load on an overloaded gateway. On the other hand, each time a node changes its next hop (for the same gateway), the channel access pattern in its locality (and eventually a large area) may be diversely affected, resulting in packet drops at the medium access control (MAC) sublayer. In fact, since nodes make independent routing decisions, the data paths in the network may become highly unstable if the nodes greedily select the best next hop to each gateway each time there is an opportunity to do so. This is particularly a problem when path performance metrics vary too frequently due to network dynamics. As a remedy, RALB monitors the following two variables over a window $\mathrm{W}$ of recent data packet arrival time instances, called the incident window for each gateway:

1) Path quality metric Difference Ratio: $D R=\left(q\left(p^{*}\right)-\right.$ $\left.q\left(p_{o}\right)\right) / q\left(p_{o}\right)$

2) Path Switch Frequency: $S F=s / W$,

where $s$ denotes the number of path switches that have occurred within the most recent incident window. 


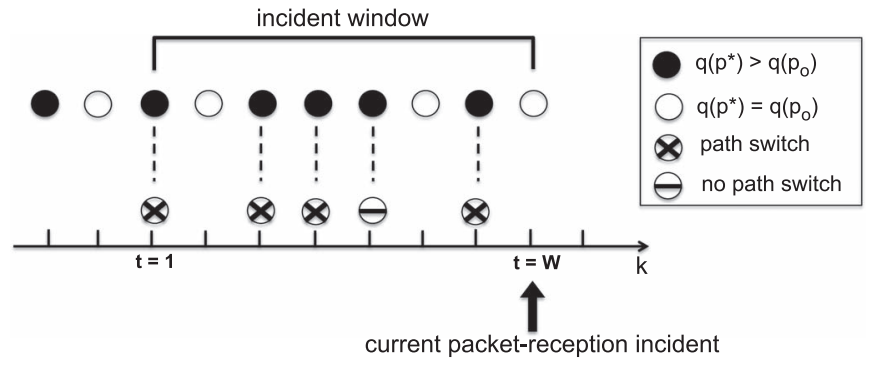

Fig. 2. Window of recent data packet reception instances.

Fig. 2 illustrates a window of the most recent $W$ instances; at each instance, a new packet to be forwarded to a gateway $g$ arrives at the node. The black dots in the top row represent the instances when the quality metric of RALB is larger than that of the shortest path, i.e., $q\left(p^{*}\right)>q\left(p_{o}\right)$ (and white dots are for $\left.q\left(p^{*}\right)=q\left(p_{o}\right)\right){ }^{5}$ RALB calculates the average difference ratio, which is denoted by $\overline{D R}=\sum_{t=1}^{W} D R(t) / n_{\text {diff }}$, where $n_{\text {diff }} \leq W$ is the number of times when $q\left(p^{*}\right)>q\left(p_{o}\right)$ within a window.

As shown in the second row of Fig. 2, out of all instances when $q\left(p^{*}\right)>q\left(p_{o}\right)$, only a subset represents the actual path switches, i.e., RALB's path $p^{*}$ is chosen instead of the shortest path $p_{o}$. This is because, RALB switches its data paths probabilistically. In other words, there is a certain probability $P(g)$ for each gateway $g$ that a node makes a path switch when the condition $q\left(p^{*}\right)>q\left(p_{o}\right)$ holds.

Instead of assigning an arbitrary value to $P(g)$, RALB dynamically trains its set of path switch probabilities, $P\left(g_{1}\right), P\left(g_{2}\right), \ldots, P\left(g_{K}\right)$, where $g_{1}, g_{2}, \ldots, g_{K} \in G$, and $G$ is the set of $K$ gateways in the network. To achieve this, RALB uses the variables $\overline{D R}$ and $S F$. Basically, the path switch probability $P(g)$ is incremented by $\Delta P$ if the most recent switch frequency $S F$ is not sufficiently high when compared with the most recent average path metric difference ratio $\overline{D R}$. Otherwise, $P(g)$ is decremented by $\Delta P$. In order to control the sensitivity of $S F$ to the changes in $\overline{D R}$, and to adapt to the path metric quality dynamics of the network, a decision threshold $T$ is introduced as follows:

\begin{tabular}{l}
\hline Algorithm 1 Update of path switch probability. \\
\hline For a gateway $g$ : \\
1: if $S F \leq \overline{D R} \cdot T$ then \\
2: $\quad P(g)=P(g)+\Delta P$ \\
3: else \\
4: $\quad P(g)=P(g)-\Delta P$ \\
5: end if \\
\hline
\end{tabular}

The logic in Alg. 1 suggests the following:

1) If a node has paths with mostly similar path quality metrics (hence a low $\overline{D R}$ ), it will have only a few occasions to increase the path switch probability $\mathrm{P}(\mathrm{g})$, hence does not perform frequent path switches,

\footnotetext{
${ }^{5}$ Please note that since RALB ranks the available paths according to their path quality metric values, and then picks the one with the highest value, the case $q\left(p^{*}\right)<q\left(p_{o}\right)$ cannot happen. Hence, $D R \neq 0$.
}

2) If the node has made many path switches recently (hence high $S F$ ), the path switch probability will be lowered so that the node's tendency to cause route flapping can be moderated when network dynamics are in effect,

3) RALB's load-balancing behavior can be tuned using the threshold $T$ : a higher value leads to more frequent path switches, whereas a lower value causes lower performance in load balancing.

Algorithm 2 The RALB algorithm: Path selection.

\section{Initialization:}

$$
k=0 ; n_{\text {diff }}=0 ; n_{\text {switch }}=0 ; D R_{\text {tot }}=0 ;
$$$$
P(g)=0 ; \Delta P=0.02 \text {; }
$$

Input from routing table:

$$
p_{o}, p^{*}, q\left(p_{o}\right), q\left(p^{*}\right)
$$

\section{At each data packet reception for gateway $g$ :}

$1: k=k+1$;

2: if $k>W$ then

3: $\quad$ window $=W$;

4: $\quad D R_{\text {tot }}=D R_{\text {tot }}-D R(1)$

5: $\quad n_{\text {switch }}=n_{\text {switch }}-S(1)$;

6: $\quad n_{\text {diff }}=n_{\text {diff }}-D(1)$;

7: else

8: $\quad$ window $=k$;

9: end if

10: if $q\left(p^{*}\right)>q\left(p_{o}\right)$ then

11: $\quad n_{\text {diff }}=n_{\text {diff }}+1$;

12: $D R=\left[q\left(p^{*}\right)-q\left(p_{o}\right)\right] / q\left(p_{o}\right)$;

13: $D R_{\mathrm{tot}}=D R_{\mathrm{tot}}+D R$;

14: $\overline{D R}=D R_{\mathrm{tot}} / n_{\mathrm{diff}}$;

15: $\quad S F=n_{\text {switch }} /$ window;

16: $\quad$ if $S F \leq \overline{D R} \cdot T$ then

17: $\quad P(g)=P(g)+\Delta P$

18: else

19: $\quad P(g)=P(g)-\Delta P$

20: end if

21: $\quad$ if $\operatorname{rand}() \leq P(g)$ then

22: $\quad n_{\text {switch }}=n_{\text {switch }}+1$;

23: $\quad p=p^{*}$;

24: else

25: $\quad p=p_{o}$;

26: end if

27: end if

28: return $p$;

RALB's path selection algorithm is presented in Alg. 2, and returns the selected path $p$ for an incoming data packet. Since RALB is fully distributed, each node runs a separate instantiation of this algorithm, upon each packet reception event. For each data flow, three arrays are used for tracking history information; each of these arrays are of length $W$, hence the storage complexity is $\mathrm{O}(W)$ per flow. The algorithm is simply a sequence of arithmetic operations, and no program loops are used; hence the time complexity is $\mathrm{O}(1)$ when the three dynamic lists are implemented as linked lists, and $\mathrm{O}(W)$ when fixed size arrays are used, as a left-shift operation is needed to update the first element in each array. These are typical 
complexity figures commonly seen in various data routing algorithms, e.g., the SBR [37] and MNC [33].

In this algorithm, each node keeps track of a discrete timeinstance counter $k$ for each destination gateway $g$. Each time a data packet to be forwarded to $g$ is received, $k$ is incremented by 1 . Initially, the window size is 1 and is updated at each algorithm run (lines 3,8). Aside from $k$, RALB also tracks the number of path switches $n_{\text {switch }}$ that have occurred and the number of times $n_{\text {diff }}$ that the path quality metrics $q\left(p_{o}\right)$ and $q\left(p^{*}\right)$ have differed within the current incident window. Path switch instances and metric difference instances are recorded in two Boolean dynamic arrays: $S$ and $D$, respectively. $D R$ values over the window are also recorded in a dynamic array, which is denoted by $D R$ (lines 4-6 in Alg. 2).

Alg. 2 updates the path switch probability as explained in Alg. 1 (line 16-20). The function $\operatorname{rand}()$ at line 21 returns a random uniform number in the range $[0,1]$. This "coin-toss" operation determines whether a path switch will take place for the current data packet. If so, the number of path switches $n_{\text {switch }}$ is incremented by 1 , and RALB's path $p^{*}$ is selected; otherwise, the default path $p_{o}$ provided by the routing algorithm is selected.

Path switches can occur only if RALB can offer a path with a higher path quality metric than that of the shortest path, i.e., $q\left(p^{*}\right)>q\left(p_{o}\right)$ (line 10). In this case, updated values of the variables $\overline{D R}$ and $S F$ are needed. To achieve this, the sum of the $D R$ values of all incident points in the current window are kept as a separate variable denoted by $D R_{\text {tot }}$ (line 13). ${ }^{6}$ Note that, as the incident window slides after reaching a size of $W$, the first incident point needs to shift one position to right as well, and is therefore no longer within the window. Hence, the $D R$ value of the window's first point, i.e., $D R(1)$, is subtracted from the total $D R_{\mathrm{tot}}$. Similarly, as the window slides to the right by one position. $n_{\text {switch }}$ and $n_{\text {diff }}$ are updated at lines 5 and 6, by subtracting $S(1), D(1)$, respectively. The next time a data packet arrives, $D(1), S(1)$, and $D R(1)$ will refer to the first position in their respective dynamic arrays. ${ }^{7}$

\section{Performance Evaluation}

Here, the performance of the proposed RALB solution is evaluated by simulations. First, the key parameters of RALB are tested in an intuitively simple topology, in which the tradeoff between multihop path length and gateway capacity is clear. After this verification step, RALB is evaluated in large-scale random network topologies. Both the proof-of-concept and the large-scale network simulations are performed using Network Simulator 2 (ns-2) [46].

\section{A. Proof-of-Concept Simulations}

As mentioned in Section III, RALB is an adaptive and iterative solution, and modifies a system variable $P(g)$ at each node. This variable controls the probability to choose

\footnotetext{
${ }^{6}$ This avoids having to perform summation operations over the array, each time a data packet arrives.

${ }^{7}$ A linked-list implementation can direct the starting point to the next position in $\mathrm{O}(1)$ time, although a full array-shift would be of low time complexity, as well, i.e., $\mathrm{O}(W)$.
}

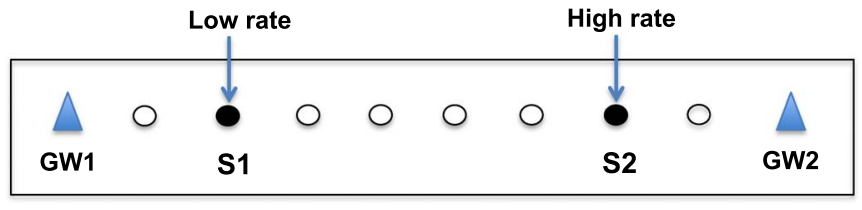

Fig. 3. Topology of the proof-of-concept simulations.

TABLE II

SiMUlation PARAMETERS

\begin{tabular}{|c|c|c|}
\hline Parameter & Proof-of-concept & Large-scale \\
\hline \hline Topology dimensions & $1000 \mathrm{~m}^{*} 100 \mathrm{~m}$ & $500 \mathrm{~m} * 200 \mathrm{~m}$ \\
\hline Wireless MAC & IEEE $802.11 \mathrm{~b}$ & IEEE $802.11 \mathrm{~b}$ \\
\hline Propagation model & Two-ray ground & Two-ray ground \\
\hline Transmission range $(m)$ & 60 & 60 \\
\hline Number of nodes & 8 & 140 \\
\hline Number of gateways & 2 & 2 \\
\hline CBR packet size $($ bytes $)$ & 512 & 512 \\
\hline CBR packet interval $(s)$ & $0.8,1.6$ & $0.4,0.5,0.6,0.7,0.8$ \\
\hline Number of source nodes & 2 & 40 \\
\hline Gateway capacity $(K b p s)$ & 6.4 & 64,256 \\
\hline Incident window & 10 & 10 \\
\hline
\end{tabular}

the path with the highest path quality metric value rather than the shortest path which is most of the time suggested by the underlying load-unaware routing protocol, when the two are different. Considering this probabilistic behavior of RALB, two extremes can be defined. First, a load-unaware routing protocol (AOMDV [16] in this paper) represents choosing the "default" path frequently, without considering the path quality metric, i.e., $P(g)=0$. The second extreme is to choose the path with the highest quality metric all the time, i.e., $P(g)=1$, which is called pure load balancing (PLB) in this paper.

Before moving on to large-scale network simulations, the first evaluation aim is to verify that RALB is indeed a middleway solution between these two extremes. To achieve this, a simple topology, as shown in Fig. 3, is considered.

In Fig. 3, two packet gateways with the same capacity, which are denoted by GW1 and GW2, serve the same purpose of receiving data packets from the two data sources, which are denoted by S1 and S2, which have different data generation rates. Each source is closer to one of the gateways, which makes that particular gateway a more intuitive and desirable option from the perspective of a load-unaware routing protocol. Since the sources have different data rates, without any loadaware solution in place, the utilizations of the two gateways are expected to be different. By applying RALB on top of AOMDV, the aim is to observe a migration of traffic from the higher loaded half of the network to the other, which corresponds to not choosing the "default" gateway. The second column in Table II summarizes the parameters of the proof-of-concept simulations.

The performance results of AOMDV, RALB, and PLB are shown in Fig. 4, which represent averages of 40 simulation runs. The figure clearly demonstrates the tradeoff between the two conflicting objectives: 1) equalization of gateway utilization values [see Fig. 4(c)]; and 2) network performance [see Fig. 4(a) and (b)]. As a greedy solution, PLB can achieve a low standard deviation in gateway utilization, with the cost of increased E2E delay and low packet delivery ratio. The other extreme, i.e., load-unaware AOMDV, has high network 


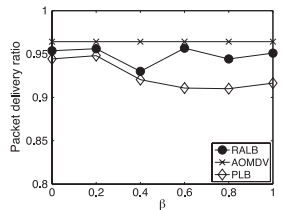

(a)

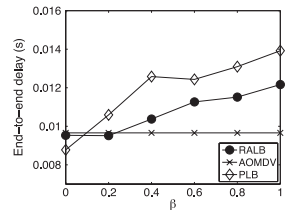

(b)

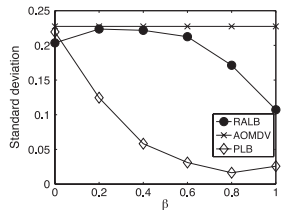

(c)
Fig. 4. Simulation results for the topology of Fig. 3. (a) Packet delivery ratio. (b) E2E delay. (c) Standard deviation of gateway utilizations.

performance, with no equalization gains. Between these two, RALB can achieve reduction of standard deviation as opposed to AOMDV, whereas showing better network performance than the greedy strategy PLB. In short, RALB is able to control the frequency of path switches with its probabilistic approach.

Fig. 4 also demonstrates the effect of the coefficient $\beta$, which controls the relative proportions of gateway utilization and path quality terms in the path metric [see (1)]. Note that only RALB and PLB consider the path metric, whereas AOMDV does not, hence AOMDV's performance is independent from $\beta$.

In (1), a high value of $\beta$ implies more emphasis on gateway utilization. This effect is observed for RALB and PLB in Fig. 4(c), which both show a decreasing standard deviation for larger $\beta$. For RALB, however, the decrease is observed to be noticeable after around $\beta=0.5$. This implies a cutoff value over which the difference in gateway utilization becomes the dominant factor when choosing between two paths. Hence, simulations verify the expected behavior from (1): the coefficient $\beta$ effectively controls the bias on achieving a better gateway utilization as opposed to choosing a path with better quality. In short, for a sufficiently small $\beta$, RALB is reluctant to sacrifice path quality for better equalization of gateway utilizations.

\section{B. Large-Scale Network Simulations}

In this part, the performance evaluation of the proposed RALB solution in randomly deployed large-scale networks is presented. RALB is compared with the following:

1) AOMDV [16], which is a load-unaware multipath reactive protocol that ranks the available data paths with respect to their $\mathrm{E} 2 \mathrm{E}$ time delay,

2) $\mathrm{MNC}+$, which is on adaptation of the maximum normalized capacity (MNC) protocol presented in a recent study [33]. MNC is proactive and is based on OLSR [47], whereas $\mathrm{MNC}+$ is reactive and runs on AOMDV. This is a measure to avoid periodic gateway advertisements of OLSR. MNC+ is load-aware, considers the following two metrics (same as MNC): the available gateway capacity and hop distance to the gateway,

3) source-based routing (SBR) (recent study in [37]), which is also load-aware, and considers the traffic loads on gateways (rather than gateway capacity) and a path metric (rather than only hop distance). SBR's path metric is a combination of expected link quality (represented as the success rate of transmitted probe packets) and interference ratio (ratio of the sum of the amounts of interference power from all interfering nodes over the maximum tolerable interference at the receiver radio). SBR defines gateway load as the average packet queue length.

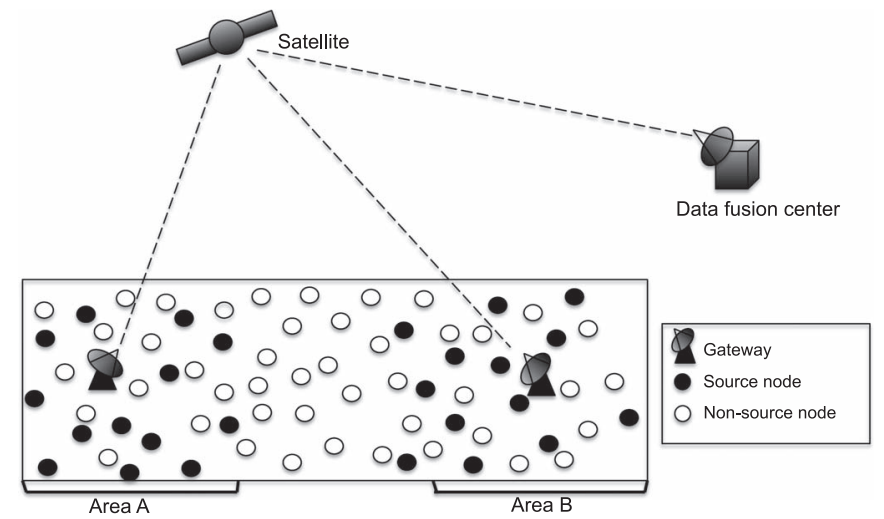

Fig. 5. Remote monitoring of two neighbor areas via satellite links.

Both RALB and MNC+ are built on top of AOMDV, with different path selection metrics. In both algorithms, the time complexity of computing the path selection metric and choosing the best path upon each reception event is $\mathrm{O}(1)$. Furthermore, the network initiation complexity of these algorithms is $\mathrm{O}(2 d)$, where $d$ is network diameter [48]. SBR is built based on table-driven protocols with a load-balancing path selection metric; hence, its time complexity is $\mathrm{O}(d)$ [48].

In performance comparisons, four metrics are considered: 1) packet delivery ratio, to observe the reliability of the routes selected by the protocols; 2) E2E time delay, as a means to measure how quickly the collected information is delivered to the infrastructure; 3) standard deviation of gateway utilization levels (as defined in Section III), to see how much load equalization is obtained among gateways, and finally, 4) control overhead, which is the total number of control packets.

Fig. 5 demonstrates the network topology that is used in performance evaluations. In this topology, two neighboring areas are remotely monitored by a data fusion center via satellite links. In each area, 20 nodes sense the environment (source nodes) and generate data streams, which are simulated as constant bit rate (CBR) packet flows with rates ranging in $[5,10] \mathrm{Kb} / \mathrm{s}$. Ku-band satellites with uplink bandwidths of 64 and $256 \mathrm{~Kb} / \mathrm{s}$ are considered [49]. Simulations are performed using Network Simulator 2 (ns-2) [46], and the third column in Table II summarizes the large-scale network simulation parameters.

\section{Simulation Scenarios}

In a network where source nodes deliver collected information to one of multiple available gateways over multihop paths, traffic load imbalance on gateways may occur due to two main reasons:

1) The data sources that would normally choose a specific gateway (due to its proximity) may generate high aggregate traffic which the gateway cannot serve; hence, some of that traffic must be diverted to another gateway if the latter is capable). This high traffic can be caused by (1) a large number of sources around the gateway, (2) high data generation rates, or (3) both,

2) Some gateways may have low capacity, whereas others are able to accommodate their current traffic loads. 
TABLE III

SimUlation SCENARIOS FOR THE TOPOLOGY IN Fig. 5

\begin{tabular}{|l||c|c|c|c|}
\hline \multirow{2}{*}{\multicolumn{1}{|c||}{ Scenario }} & \multicolumn{2}{c|}{$\begin{array}{c}\text { Number of } \\
\text { source nodes }\end{array}$} & \multicolumn{2}{c|}{$\begin{array}{c}\text { Gateway } \\
\text { bandwidth }(\mathrm{Kbps})\end{array}$} \\
\cline { 2 - 5 } & Area A & Area B & Area A & Area B \\
\hline 1. Heterogeneous gateways & 20 & 20 & 64 & 256 \\
\hline 2. Heterogeneous sources & 30 & 20 & 256 & 256 \\
\hline 3. Homogeneous & 20 & 20 & 256 & 256 \\
\hline
\end{tabular}

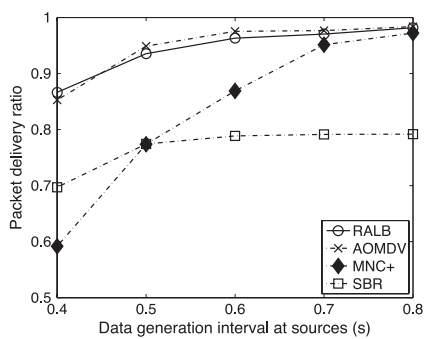

(a)

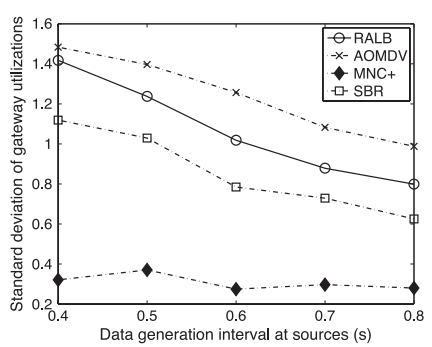

(c)

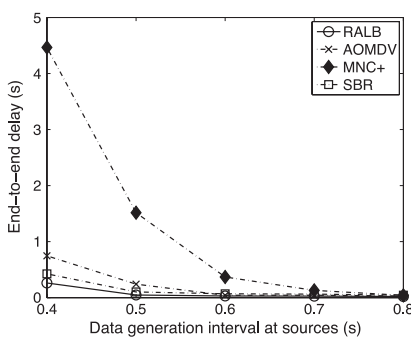

(b)

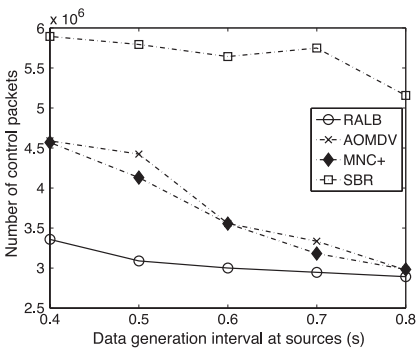

(d)
Fig. 6. Scenario 1: Heterogeneous gateways. (a) Packet delivery ratio. (b) E2E delay. (c) Gateway utilization stability. (d) Number of control packets.

Considering these two factors that may cause traffic imbalance, three scenarios are studied in performance evaluations ${ }^{8}$ : 1) Heterogeneous gateways, with different capacities represented by the bandwidth on the satellite link;2) heterogeneity of data sources, in which one of the areas have more data sources; and 3) homogeneous conditions. The common aim in these different simulation scenarios is to show that, as opposed to $\mathrm{MNC}+$ and SBR, the adaptive and probabilistic path-switch strategy of RALB is effective in migrating the traffic flows to equalize gateway utilizations, while not hindering network performance considerably. The parameters for simulation scenarios are shown in Table III.

1) Scenario 1 (Heterogeneous Gateways): In this scenario, the capacities of the two gateways are different. Fig. 6 shows the performance results. Compared with AOMDV, RALB achieves reduction in the standard deviation of gateway utilizations [see Fig. 6(c)], hence load balancing, while ensuring that the level of reliability is still comparable to that provided by AOMDV [see Fig. 6(a)]. The E2E delay is also improved [see Fig. 6(b)] for low values of the data packet generation time interval at sources (high rates of data packet generation). In such cases, one of the gateways gets overloaded, resulting in retransmissions and an eventual higher average E2E delay. RALB can reduce this effect by diverting some of the traffic flows to the alternative gateway. Similar reduction is achieved by SBR. However, one

\footnotetext{
${ }^{8}$ The sum of all gateway capacities is larger than the total generated data traffic rate; otherwise it is inevitable for at least one gateway to be overloaded.
}

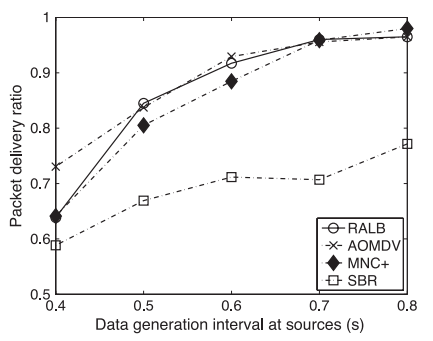

(a)

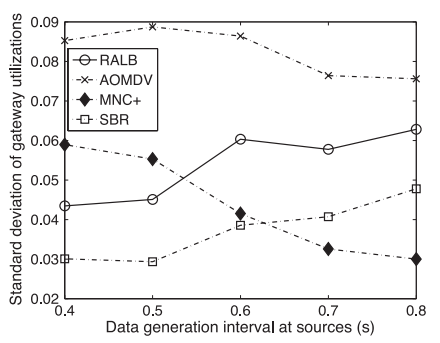

(c)



(b)



(d)
Fig. 7. Scenario 2: Different average data generation rates at the two network areas, i.e., areas A and B, as depicted in Fig. 5. (a) Delivery ratio. (b) E2E delay. (c) Gateway utilization. (d) Number of control packets.

striking observation is that RALB can reduce the number of control packets by up to around 25\% compared with AOMDV [see Fig. 6(d)]. This is attributed to the fact that paths chosen by RALB are more stable, since RALB's path quality metric also considers the reliability and residual bandwidth of each path, in addition, the E2E delay (AOMDV prioritizes paths according to E2E delay only). Hence, the necessary path discovery attempts are not as frequent as in AOMDV, resulting in lower control overhead.

$\mathrm{MNC}+$ has the best gateway utilization performance, however, with the sacrifice of the network's operational performance, i.e., poor packet delivery ratio and E2E delay. MNC+'s metric overly emphasizes the difference in gateway capacity, resulting in a greedy load-balancing performance and a high control overhead due to its periodic gateway advertisements [see Fig. 6(d)].

2) Scenario 2 (Heterogeneity in Data Sources): This scenario also introduces an inequality between the two areas $\mathrm{A}$ and B in Fig. 5. The difference is, the inequality now is caused by traffic rates but not gateway capacities. The data sources in one of the areas in Scenario 2 inject their data traffic to the network more aggressively than those in the other area. Fig. 7 demonstrates the performance results.

Similar to Scenario 1, RALB provides gains in gateway utilization equalization [see Fig. 7(c)] and E2E delay [see Fig. 7(b)], while carefully preserving similar reliability levels to AOMDV, whereas MNC+ shows loss in packet delivery ratio. Compared with RALB and MNC+, SBR has significant loss in packet delivery ratio [see Fig. 7(a)] and considerable control overhead [see Fig. 7(d)], as it reacts aggressively to the traffic load imbalance between the two areas (causing load imbalance between the gateways). MNC + in general has the highest E2E delay, although not as critical as in Scenario 1, a better reliability performance [see Fig. 7(a)], and again similar control overhead to AOMDV as in Scenario 1 [see Fig. 7(d)]. 


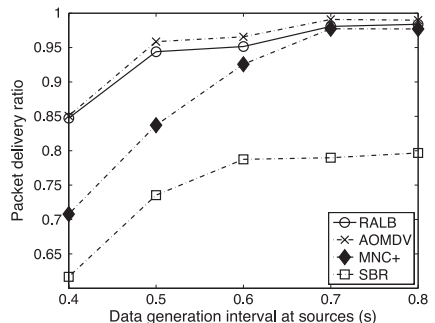

(a)

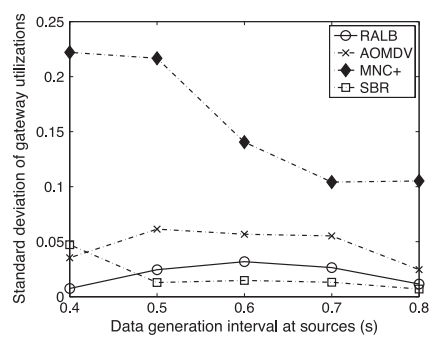

(c)

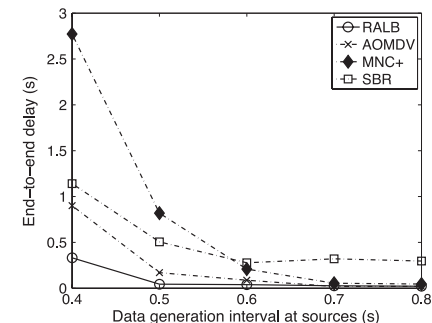

(b)

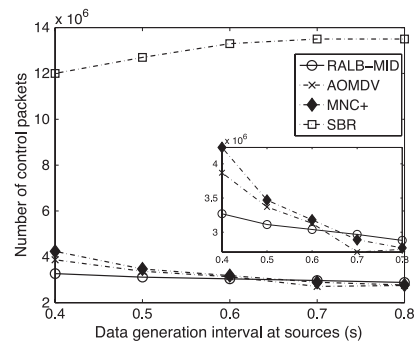

(d)
Fig. 8. Scenario 3: Homogeneous conditions. (a) Delivery ratio. (b) E2E delay. (c) Gateway utilization. (d) Number of control packets.

Fig. 7(c) is a demonstration of the differences in metric behaviors of the three load-aware solutions. MNC + is inversely affected by the change in packet generation rate [see Fig. 7(c)], as opposed to RALB and SBR. At high data rates, the residual capacity of the gateways are both low, causing hop distance to be the dominant factor in MNC+'s path selection metric. Hence, shorter paths are preferred, which on the average provide less packet delivery delay. As the traffic rate decreases, the residual gateway capacity becomes a more important factor in MNC+'s metric, and the protocol behaves like PLB, i.e., a greedy load-balancing target. In contrast, SBR and RALB become more aggressive with increasing traffic rates, resulting in better equalization in gateway utilization [see Fig. 7(c)].

3) Scenario 3 (Homogeneous Conditions): Finally, this scenario represents the conditions when there is no difference in the traffic loads of the two areas in Fig. 5, and the gateway capacities are the same. An effective routing solution is expected to not divert the traffic flows between the two areas, except local congestion issues caused by random deployment of the source nodes. Fig. 8 demonstrates the simulation results. The constructive effect of RALB over AOMDV is observed in terms of improvement in E2E delay (for high rates, when congestion is more likely) and load balancing (as it helps migrate only some of the flows, which may experience low quality paths due to the randomness in the topology). By adapting its level of aggressiveness dynamically and in a distributed way, RALB avoids making instantaneous decisions and excessive path switches. This makes RALB less vulnerable to route flapping effects, i.e., frequent changes in route choices caused by the dynamicity in path metrics, which is a result of the temporal variations in incoming traffic flow rates at network nodes and fluctuations in gateway traffic loads. Furthermore, the inset figure in Fig. 8(d) demonstrates that RALB's control overhead does not exponentially increase with the increase in the average data generation rate, unlike the others, making it a scalable solution to data rate changes.



Fig. 9. Simulation-based classification of RALB's modes of operation.

\section{Controlling the Aggressiveness of RALB: The Threshold T}

With Alg. 2 RALB controls its path switch aggressiveness, by monitoring the rate of the recent path switches (SF) and the recent average difference ratio $(\overline{D R})$ between the qualities of the default path and RALB's choice of path. A large value of the threshold $T$ in Alg. 2 permits more frequent switches, whereas a small value is more restrictive. In the last set of simulations, RALB's behavior is categorized into three classes, according to its choice of $T$, namely RALB-LOW, RALBMID, and RALB-HIGH, which correspond to conservative, moderate, and aggressive modes of operation. Based on these modes, the performance of RALB is evaluated, and Scenario 1 with heterogeneous gateways is considered.

Fig. 9 demonstrates the relation between $T$ and the resulting rate of path switch observed in RALB. The three modes of operation are designated by the equilength regions in the vertical axis, i.e., the average rate of path switch. In this figure, although $T$ is a parameter of only RALB, results for AOMDV, MNC+, and SBR are also provided as a reference. Note that AOMDV has a nonzero value. This is caused by the diversion of some traffic flows originating from locations closer to the middle of the network in Fig. 5. For such flows, AOMDV may choose the farther gateway, when there is congestion that adversely affects time delay in packet delivery. AOMDV is the most conservative protocol, since it mostly selects the shortest paths. Fig. 9 also shows that MNC + is quite aggressive. This is because, for Scenario 1 (heterogeneous gateways), the dominant factor in MNC's path metric is the residual gateway capacity, which converges its behavior to PLB (see Section IV-A). In contrast, SBR is moderate, as it has a dual target of balancing both in-network conditions and gateway loads. The difference of RALB is its adaptive and iterative approach, which presents a monotonically increasing level of aggressiveness in its loadbalancing behavior, with respect to its parameter $T$.

According to the classification in Fig. 9, the threshold is set as $T=0.2,0.6,0.9$ for RALB-LOW, RALB-MID, and RALB-HIGH modes, respectively. Fig. 10 demonstrates the performance results for these different modes of operation. This figure also shows the tradeoff between load-balancing and network QoS conditions. The highest gains with respect to the load-unaware AOMDV are obtained for higher data generation 


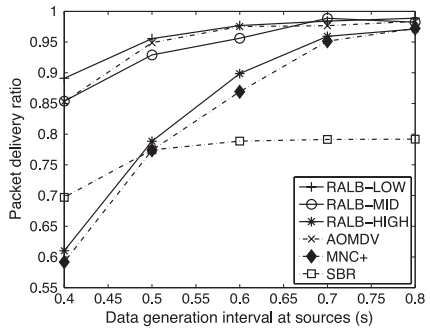

(a)

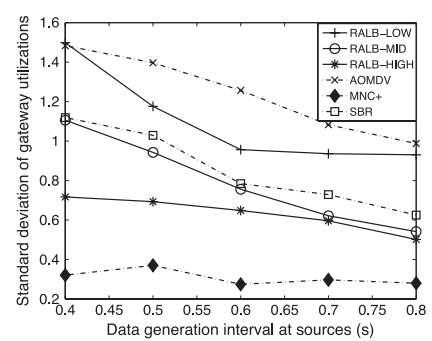

(c)



(b)

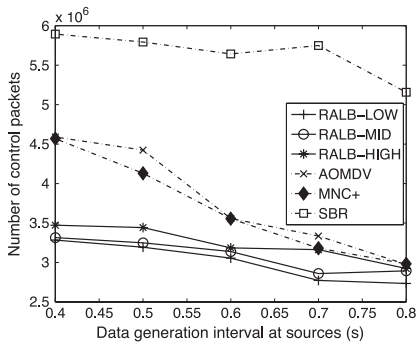

(d)
Fig. 10. Performance comparison of different mode of RALB. (a) Delivery ratio. (b) E2E delay. (c) Gateway utilization equalization. (d) Number of control packets.

rates (lower data generation interval), where congestion in the network and load imbalance on the gateways become more apparent, and the benefits of an adaptive load-balancing solution are better observed. An aggressive path switch policy, such as $\mathrm{MNC}+$, can achieve high gateway load-balancing performance [see Fig. 10(a)], with the cost of degrading the in-network conditions [see Fig. 10(a) and (b)].

Among the three RALB modes, the aggressive RALB-HIGH achieves the best load-balancing performance [see Fig. 10(c)], however, with a cost of increased E2E delay and low packet delivery ratio. In contrast, the conservative RALB-LOW can deliver similar reliability guarantees as AOMDV, while lowering both gateway utilization difference and E2E delay in packet delivery. As expected, RALB-MID has a moderate performance in between these two.

Fig. 10(d) shows that all three RALB modes have the least control overhead, as compared with others; a clear benefit of RALB's path quality metric which picks more reliable paths. This is in contrast to AOMDV and MNC+ which consider merely path latency when ranking the available paths. Furthermore, RALB stores multiple paths in its node routing tables, whereas AOMDV replaces the existing paths with the newly discovered ones. Thus, it is more likely to reinitiate path discovery in AOMDV than in RALB. Compared with others, the higher control overhead of SBR stems from its proactive path discovery mechanism. As a result, even if SBR has a moderate rate of path switch (Fig. 9), it suffers from its periodic operation. In contrast, the AOMDV-based reactive $\mathrm{MNC}+$ has a lower control overhead.

\section{CONCLUSiON}

In this paper, RALB is proposed for data collection in environmental remote monitoring applications through a WSN to a group of satellite platforms. The main challenge in such applications is the limited bandwidth on the satellite link, which may easily become a communications bottleneck at times when the delivered data volumes to different gateways become disproportionate, due to the random nature of WSN deployments and when environmental events occur at specific parts of the network. Furthermore, in-network traffic congestion should also be avoided. RALB addresses this dual objective of gateway and in-network load balancing. It combines multiple path metrics (path residual bandwidth, E2E delay and path reliability) as well as gateway conditions (gateway utilization) in a unified path quality metric, in order to accurately account for these factors when ranking multiple available paths. RALB is designed to probabilistically choose an alternative path, instead of the shortest path that is often selected by multipath reactive routing protocols. The solution adaptively and iteratively modifies its path switch probability by means of independent decisions made by network nodes. This distributive property makes RALB a scalable solution, which is also adaptive to the latest path conditions, with minimal control overhead.

Extensive simulation results using the ns-2 simulator on randomly deployed large-scale networks demonstrate the effectiveness of the proposed algorithm: RALB can reduce the difference in the utilizations of multiple available data gateways that act as the network's data sinks, and improves network performance by avoiding less qualified data paths, which provides less E2E delay in packet delivery and comparable packet delivery ratio to AOMDV. RALB is also shown to reduce control overhead of AOMDV over a practical range of source data generation rates, which makes it a suitable solution for load-balancing in wireless sensor networks.

\section{REFERENCES}

[1] M. Li, Y. Liu, and L. Chen, "Non-threshold based event detection for 3D environment monitoring in sensor networks," IEEE Trans. Knowl. Data Eng., vol. 20, no. 12, pp. 1699-1711, Dec. 2008.

[2] K. Akkaya and M. Younis, "A survey on routing protocols for wireless sensor networks," Ad Hoc Netw., vol. 3, no. 3, pp. 325-349, May 2005.

[3] S. H. Lee, S. Lee, H. Song, and H. S. Lee, "Wireless sensor network design for tactical military applications: Remote large-scale environments," in Proc. IEEE MILCOM, Oct. 2009, pp. 1-7.

[4] S.-Y. Cheung and P. Varaiya, "Traffic surveillance by wireless sensor networks: Final report," University of California, Berkeley, Berkeley, CA, USA, California PATH Res. Rep. UCB-ITS-PRR-2007-4, 2007.

[5] C.-Y. Chong and S. P. Kumar, "Sensor networks: Evolution, opportunities, and challenges," Proc. IEEE, vol. 91, no. 8, pp. 1247-1256, Aug. 2003.

[6] Cool It! Space Apps Challenge. accessed: 2015-05. [Online]. Available: https://2014.spaceappschallenge.org/challenge/cool-it/

[7] DREAMSys: Distributed Remote Environmental Array and Monitoring System. acc'ed: 2015-05. [Online]. Available: http://projects.npl.co.uk/ dreamsys/index.html

[8] SENSEIVER. accessed: 2015-05. [Online]. Available: http://senseiver. com/

[9] ATWARM: Advanced Technologies for Water Resource Management. accessed: 2015-05. [Online]. Available: http://www.qub.ac.uk/researchcentres/ATWARM/

[10] MONET: Mechanisms for Optimization of Hybrid Ad-Hoc Networks and Satellite Networks. accessed: 2015-05. [Online]. Available: http://monet. tekever.com/home

[11] I. Mathioudakis, N. M. White, N. R. Harris, and G. V. Merrett, "Wireless sensor networks: A case study for energy efficient environmental monitoring," in Proc. Eurosensors, Dresden, Germany, Sep. 2008.

[12] ARGOs System. accessed: 2015-05. [Online]. Available: http://www. argos-system.org/en/

[13] N. Celandroni et al., "A survey of architectures and scenarios in satellitebased wireless sensor networks: System design aspects," Int. J. Satell. Commun. Netw., vol. 31, no. 1, pp. 1-38, Jan./Feb. 2013.

[14] S. Verma, P. Pillai, and Y.-F. Hu, "Performance evaluation of alternative network architectures for sensor-satellite integrated networks," in Proc. WAINA, Mar. 2013, pp. 120-125. 
[15] Y. Xue, B. Ramamurthy, and M. Burbach, "A two-tier wireless sensor network infrastructure for large-scale real-time groundwater monitoring," in Proc. IEEE LCN, Oct. 2010, pp. 874-881.

[16] M. K. Marina and S. R. Das, "Ad hoc on-demand multipath distance vector routing," SIGMOBILE Mobile Comput. Commun. Rev., vol. 6, no. 3, pp. 92-93, Jul. 2002.

[17] K. Tada and M. Yamamoto, "Load-balancing gateway selection method in multi-hop wireless networks," in Proc. IEEE GLOBECOM, 2009, pp. 1-6.

[18] J. J. Galvez, P. M. Ruiz, and A. F. Gómez-Skarmeta, "Responsive online gateway load-balancing for wireless mesh networks," Ad Hoc Netw., vol. 10, no. 1, pp. 46-61, Jan. 2012.

[19] S. Avallone and G. D. Stasi, "A new MPLS-based forwarding paradigm for multi-radio wireless mesh networks," IEEE Trans. Wireless Commun., vol. 12, no. 8, pp. 3968-3979, Aug. 2013.

[20] G. L. Liao, C. Y. Chen, S. W. Hsu, and T. Y. Wu, "Adaptive situationaware load balance scheme for mobile wireless mesh networks," in Proc. IEEE INFOCOM WKSHPS, 2011, pp. 66-74.

[21] J. Broch, D. A. Maltz, D. B. Johnson, Y.-C. Hu, and J. Jetcheva, "A performance comparison of multi-hop wireless ad hoc network routing protocols," in Proc. ACM MobiCom, 1998, pp. 85-97.

[22] T. He, J. A. Stankovic, C. Lu, and T. F. Abdelzaher, "A spatio-temporal communication protocol for wireless sensor networks," IEEE Trans. Parallel Distrib. Syst., vol. 16, no. 10, pp. 995-1006, Oct. 2005.

[23] S. Avallone, G. D. Stasi, and A. Kassler, "A traffic-aware channel and rate reassignment algorithm for wireless mesh networks," IEEE Trans. Mobile Comput., vol. 12, no. 7, pp. 1335-1348, Jul. 2013.

[24] V. Mhatre, H. Lundgren, F. Baccelli, and C. Diot, "Joint MAC-aware routing and load balancing in mesh networks," in Proc. CoNEXT, 2007, pp. 19:1-19:12.

[25] V. Mhatre, H. Lundgren, and C. Diot, "MAC-aware routing in wireless mesh networks," in Proc. WONS, 2007, pp. 46-49.

[26] Y. Fu, K.-M. Chan, K. S. Tan, and B. S. Yeo, "Multi-metric gateway discovery for MANET," in Proc. IEEE VTC Spring, 2006, pp. 777-781.

[27] S. H. Bouk, I. Sasase, S. H. Ahmed, and N. Javaid, "Gateway discovery algorithm based on multiple QoS path parameters between mobile node and gateway node," J. Commun. Netw., vol. 14, no. 4, pp. 434-442, Aug. 2012.

[28] S. Jung, M. Kserawi, D. Lee, and J.-K. K. Rhee, "Distributed potential field based routing and autonomous load balancing for wireless mesh networks," IEEE Commun. Lett., vol. 13, no. 6, pp. 429-431, Jun. 2009.

[29] K. Ramachandran, I. Sheriff, E. Belding, and K. Almeroth, "Routing stability in static wireless mesh networks," in Proc. 8th Int. Conf. PAM, 2007, pp. 73-83.

[30] Y. Miao, Z. Sun, F. Yao, N. Wang, and H. S. Cruickshank, "Study on research challenges and optimization for internetworking of hybrid MANET and satellite networks," in PSATS, 2013, pp. 90-101.

[31] C.-F. Huang, H.-W. Lee, and Y.-C. Tseng, "A two-tier heterogeneous mobile ad hoc network architecture and its load-balance routing problem," in Proc. MONET, 2004, vol. 9, pp. 379-391.

[32] V. Pham, E. Larsen, O. Kure, and P. E. Engelstad, "Gateway load balancing in future tactical networks," in Proc. IEEE Mil. Commun. Conf., 2010 pp. $1844-1850$.

[33] E. Ancillotti, R. Bruno, and M. Conti, "Load-balanced routing and gateway selection in wireless mesh networks: Design, implementation and experimentation," in Proc. IEEE WOWMOM, 2010, pp. 1-7.

[34] D. S. Nandiraju, L. Santhanam, N. Nandiraju, and D. P. Agrawal, "Achieving load balancing in wireless mesh networks through multiple gateways," in Proc. IEEE MASS, 2006, pp. 807-812.

[35] J. Shin, J. Wang, and S. Kim, "Load balancing among Internet gateways in ad hoc networks," in Proc. IEEE VTC Fall, 2005, pp. 1677-1680.

[36] U. Ashraf, S. Abdellatif, and G. Juanole, "Gateway selection in backbone wireless mesh networks," in Proc. IEEE WCNC, 2009, pp. 2548-2553.

[37] M. Boushaba, A. Hafid, and M. Gendreau, "Source-based routing in wireless mesh networks," IEEE Syst. J., vol. 1, pp. 1-9, 2014.

[38] Q. Le-Trung, P. E. Engelstad, T. Skeie, and A. Taherkordi, "Load-balance of intra/inter-MANET traffic over multiple Internet gateways," in Proc. ACM MoMM, 2008, pp. 50-57.

[39] V. Pham, E. Larsen, P. E. Engelstad, and I. Kure, "Performance analysis of gateway load balancing in ad hoc networks with random topologies," in Proc. ACM MOBIWAC, 2009, pp. 66-74.

[40] C. Perkins et al., "Ad-hoc on-demand distance vector routing," in Proc. IEEE WMCSA, 1999, pp. 90-100.

[41] D. B. Johnson, D. A. Maltz, and J. Broch, "DSR: The dynamic source routing protocol for multi-hop wireless ad hoc networks," in Ad Hoc Networking. Reading, MA, USA: Addison-Wesley, 2001, pp. 139-172.

[42] Zigbee Alliance. accessed: 09/2014. [Online]. Available: http://www. zigbee.org/

[43] F. Cuomo, S. D. Luna, U. Monaco, and T. Melodia, "Routing in ZigBee: Benefits from exploiting the IEEE 802.15.4 association tree," in Proc. IEEE ICC, 2007, pp. 3271-3276.
[44] N. E. Rachkidy, A. Guitton, and C. Buratti, "Improving the AODV-based ZigBee routing protocol through pivots," in Proc. 24th IEEE Annu. Int. Symp. PIMRC, 2013, pp. 1983-1988.

[45] A. Gudipati and S. Katti, "Strider: Automatic rate adaptation and collision handling," in Proc. ACM SIGCOMM, 2011, pp. 158-169.

[46] The Network Simulator 2 (ns-2). accessed: 08/2014. [Online]. Available: http://www.isi.edu/nsnam/ns/

[47] P. Jacquet et al., "Optimized link state routing protocol for ad hoc networks," in Proc. IEEE INMIC, 2001, pp. 62-68.

[48] E. Royer and C.-K. Toh, "A review of current routing protocols for ad hoc mobile wireless networks," IEEE Pers. Commun., vol. 6, no. 2, pp. 46-55, Apr. 1999.

[49] H. Tripp and A. Ford, "Understanding satellite broadband quality of experience-Final report," Ofcom, London, U.K., Ofcom Rep. 72/11/R/193/R, 2011.

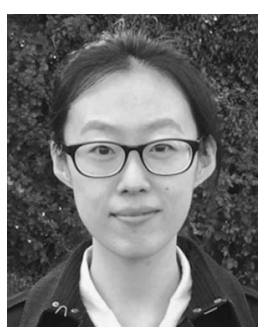

Ye Miao received the B.Sc. degree in electronic information science and technology from China Agricultural University, Beijing, China, in 2010 and the M.Sc. degree in mobile and satellite communication from University of Surrey, Surrey, U.K., in 2011. She is working toward the Ph.D. degree in the 5G Innovation Centre, Institute for Communication Systems, University of Surrey.

Her research interests are quality of service and routing solutions in mobile ad hoc networks (MANETs), integrated MANET-Satellite networks and wireless sensor networks.

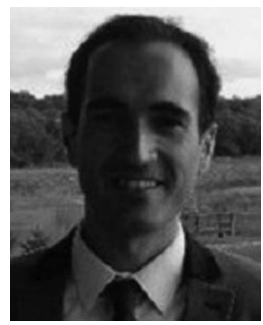

Serdar Vural received the M.S. and Ph.D. degrees in electrical and computer engineering from The Ohio State University, Columbus, OH, USA, in 2005 and 2007, respectively, and the B.S. Degree in electrical and electronics engineering from Boğaziçi University, Istanbul, Turkey, in 2003.

$\mathrm{He}$ is currently a Research Fellow with the $5 \mathrm{G}$ Innovation Centre, Institute for Communication Systems, University of Surrey, Surrey, U.K. His research interests are in wireless sensor, ad hoc, and mesh networks, Internet-of-things, machine-to-machine communications, and future internet

Dr. Vural has served as a Review Editor of IEEE COMMUNICATIONS LETTERS from October 2013 to October 2014, as a TPC member of various conferences, such as IEEE ICDCS, VTC, Globecom, WCNC, PIMRC, and as a Technical Reviewer of IEEE journals.

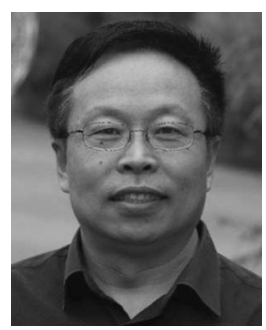

Zhili Sun received the B.Sc. degree in mathematics from Nanjing University, Nanjing, China, in 1982, and the Ph.D. degree in computer science from Lancaster University, Lancaster, U.K., in 1991.

From 1989 to 1993, he worked as a Postdoctoral Research Fellow with Queen Mary University, London, U. K. He has been Principal investigator and Technical Co-coordinator in many projects within the EU framework programs, ESA, EPSRC, and industries, and published over 125 papers in international journals, book chapters, and conferences. He is the Chair of communication networking and a Professor with the 5G Innovation Centre, Institute of Communication Systems, University of Surrey, Surrey, U.K. His research interests include wireless and sensor networks, satellite communications, mobile operating systems, traffic engineering, Internet protocols and architecture, quality of service, multicast, and security.



Ning Wang received the B.Eng. (with honors) degree from Changchun University of Science and Technology, Changchun, China, in 1996, the M.Eng. degree from Nanyang Technological University, Singapore, in 2000, and the Ph.D. degree from University of Surrey, Surrey, U.K., in 2004, respectively.

$\mathrm{He}$ is a Senior Lecturer with the 5G Innovation Centre, Institute for Communication Systems (ICS), University of Surrey. His research interests mainly include information centric networking, content and data caching management, network optimization techniques, and quality of service. 